\title{
Effects of Childhood Abuse on Major Depressive Disorder
}

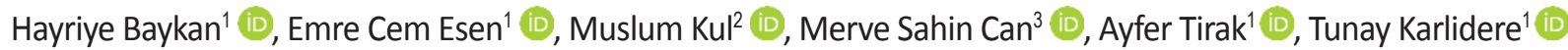 \\ ${ }^{1}$ Balıkesir University, School of Medicine, Department of Psychiatry, Balıkesir, Turkey \\ ${ }^{2}$ Mersin City Hospital, Department of Child and Adolescent Psychiatry, Mersin, Turkey \\ ${ }^{3}$ Hopa State Hospital, Department of Psychiatry, Artvin, Turkey \\ Correspondence Author: Hayriye Baykan \\ E-mail: hayriyebaykan@gmail.com
}

Received: 18.01.2018 Accepted: 19.03.2018

\begin{abstract}
Objective: Childhood trauma is a significant risk factor for major depressive disorder, which has a multifactorial etiology. We investigated the effects of childhood trauma, especially physical and sexual abuse, on depression later in adult life.

Methods: The study included 87 patients with depression and 87 healthy volunteers. Participants were asked to fill out the Childhood Trauma Questionnaire (СTQ) and Hamilton Rating Scale for Depression and Anxiety. We have used the three childhood trauma pattern groups by their CTQ scores. The first group consisted participants who did not report any childhood abuse. The second group comprised participants who had experienced emotional abuse and emotional or physical neglect. The third group included participants who reported physical and sexual abuse. Results: Approximately half of pattern groups 1 and 2 consisted of healthy controls. However, all of the participants who were in pattern group 3 had major depressive disorder. Anxiety and depression levels both had positive correlations with emotional, physical, and sexual abuse. There was a statistically significant difference between pattern groups 2 and 3 in all of the five categories of CTQ.

Conclusion: In our study, we found that incidences of physical and sexual abuse increase the risk of depression. Furthermore, they indicate an increased risk for a relationship that consists of emotional neglect and abuse, which is an independent risk factor for major depressive disorder. Therefore, we should not only focus on the individual traumas on childhood trauma cases but also consider if there is a defective pattern of relationship.
\end{abstract}

Keywords: Trauma, Major depressive disorder, Anxiety disorder, Sexual abuse, Physical abuse

\section{INTRODUCTION}

According to the World Health Organization (WHO), major depressive disorder is the second most debilitating disease (1). Epidemiological studies in Turkey show that approximately $80 \%$ of the population suffers from major depressive disorder (2). Moreover, only a quarter of those patients receive adequate treatment (2). Childhood trauma is a significant risk factor for major depressive disorder, which has a multifactorial etiology (3).

Trauma is a deeply distressing or disturbing experience. Even if it caused clinical consequences or not, every individual who ever existed experienced trauma at some point in their life. However, childhood trauma is dissimilar to the ones experienced in older ages $(4,5)$. The naive and helpless nature of minors makes them more susceptible, but it is not the only contrast they have with adults.

In adult life, conditions, such as acute stress disorder, posttraumatic stress disorder, and major depressive disorder, are associated with stress and trauma (6). Most of the time, these psychopathologies are limited to traumatic incidences. Psychopathology usually disappears when the experience is resolved (6). However, it is a very different story if it occurs during childhood. Trauma during the early years of life also impairs neurodevelopmental and psychological progress $(7,8)$. Moreover, it is debated to be the cause of personality disorders (9).

In the 1983 International Conference on Psychological Abuse of Children and Youth, abuse and neglect were investigated and thoroughly debated (10). Various classifications for abuse and neglect of children were brought forward since then. Emotional abuse, emotional neglect, physical abuse, physical neglect, and sexual abuse are the broad but distinct classifications of childhood abuse and neglect, which also are the five main categories in the Childhood Trauma Questionnaire (CTQ) (10-12).

According to WHO, child abuse means to harm a child's health, impair their sense of trust and responsibility, and compromise the process of their maturation. A child getting hurt physically in any other way than an accident is physical abuse (13). The frequency of physical maltreatment varies a lot with culture, and it is hard to collect precise data on the subject. Slapping and smacking are common discipline methods, especially in Eastern cultures. For example, in the United States, the rate of occurrence of physical abuse varies widely between $4 \%$ and $85 \%$, and in Eastern countries, such 
as Egypt or India, it is estimated to be between a quarter and three quarters (14). Moreover, the rates do not only vary with culture but also vary with socioeconomic status as well. Studies established that the risk of experiencing child abuse is higher than double in lower socioeconomic families (15).

Just like other kinds of abuses, most of the childhood experiences of sexual violence are caused by close relatives (16). This situation is one of the reasons why childhood sexual abuse is under-reported. In Turkey, less than a sixth of the sexual abuse cases are being reported (16). It makes it harder to detect the victim, criminally charge the perpetrator, and, in some cases, causes the sexual abuse to prolong if a case goes unreported. It has been reported that $9 \%$ to $18 \%$ of the pre-adolescent population in Turkey have experienced sexual injury (17). Even though the frequency of physical molestation is higher in the lower socioeconomic community, the rates of sexual abuse remain mostly the same (15).

On the other hand, neglect is a term used for not providing the sufficient necessities of a child, such as a shelter, education, healthcare, and compassion, by their parents or their legal guardians (7). As opposed to abuse, the definition of neglect has fuzzy edges. Actions, and in some cases inactions, which can be considered emotional neglect, vary with cultural and social norms (10).

According to a systematic review, childhood abuse and neglect cause an increase in psychiatric disorders, drug uses, suicide attempts, and risky sexual behaviors (18). However, the aftermath of childhood trauma is not limited to mental illnesses, and conditions, such as cardiovascular diseases, cancer, and autoimmune diseases, have been also associated with childhood trauma (19).

In the present study, we investigated the effects of childhood trauma on depression later in adult life in a Turkish population. Our hypothesis was that children who experienced childhood trauma, primarily physical and sexual abuse, are more susceptible to depression.

\section{METHODS}

\subsection{Subjects}

This was a case-control study design. The study group included 87 patients with major depressive disorder. All patients with major depressive disorder who were admitted to the outpatient clinic of Psychiatry Balıkesir University between September 2015 and August 2016, who were receiving such diagnosis for the first time in their lives, who were not taking any psychotropic medication, and who have agreed to participate were enrolled in our study. The control group comprised 87 healthy volunteers who were matched in age and gender with the study group. Healthy controls were selected among the people who work in our institution and their relatives. All participants were examined and assessed according to the Structured Clinical Interview for Diagnostic and Statistical Manual of Mental Disorders, 4th Edition (DSM-IV) Axis I disorders (SCID-1) by psychiatrists in our outpatient service. Study group participants were selected among patients who were diagnosed with major depressive disorder according to DSM-IV. Control group participants were not diagnosed with any psychiatric diseases after the SCID-1 assessment.

Participants of the study were examined by a physician, and their written medical history in our hospital and self-report medical history were investigated. Participants in both the study and the control groups were excluded if they had a medical history of delirium, dementia, psychotic disorders, bipolar disorder, or any other psychiatric disorders; mental retardation; and infectious disorders or with any other chronic diseases. Patients with depressive disorder or healthy volunteers who were pregnant were not admitted to the study. SCID-1 was applied to all our participants by psychiatrists in our outpatient service, and major depressive disorder was diagnosed in the participants in the study group after SCID-1 was applied.

Participants were asked to fill out sociodemographic data forms, and an informative consent form was given. CTQ, Hamilton Rating Scale for Depression (HAM-D), and Hamilton Rating Scale for Anxiety (HAM-A) were all applied to the participants by our clinic's psychologist. The local ethics committee approved the study. Informed consent was obtained from all the participants in the study.

\subsection{CTQ}

CTQ evaluates emotional abuse, emotional neglect, physical abuse, physical neglect, and sexual abuse that a participant has experienced during childhood years. The questionnaire is composed of 28 Likert-type items providing an assessment between 1 and $5 ; 1$, never and 5, usually. Each of the five classifications, emotional abuse, emotional neglect, physical abuse, physical neglect, and sexual abuse, have five items assigned to their category; therefore, the least score a group can obtain is $5(11,12)$.

In the validity and reliability study of the Turkish version of CTQ, any score $>5$ is suggested to be considered as positive for physical and sexual abuse categories. The same study indicates that 8 should be the cut-off score for emotional and physical neglect and 13 for emotional neglect (11).

On the other hand, these five different categories of childhood trauma usually are not in isolation from each other, and cases of solitary traumas are sparse. We cannot comprehend the cumulative effect of abuse and neglect if we investigate trauma as the sum of isolated incidents but a pattern of relationship between the abuser and the child (6).

Therefore, we have used the three childhood trauma pattern groups suggested by Schilling et al. (19). The first group consisted of participants with no childhood abuse (emotional abuse $\leq 7$, emotional neglect $\leq 12$, physical abuse $=5$, physical neglect $\leq 7$, and sexual abuse $=5$; all the criteria mentioned above have to be met for a participant to enroll into this group). The second group consisted of participants who 
have experienced noteworthy emotional abuse, emotional neglect, and/or physical neglect. However, the participants in the second group encountered mild to no physical and sexual abuse ( $5<$ physical abuse $<10$ and $5<$ sexual abuse $<8$, both of these criteria have to be met; one or more of the following criteria have to be met for emotional abuse $>7$, emotional neglect $>12$, and physical neglect $>7$ ). The third group consisted of participants who have experienced significant physical and sexual abuse (sexual abuse $\geq 8$ and physical abuse $\geq 10$, any of the two criteria have to be met).

\subsection{Anxiety and depression scales}

The Turkish versions of HAM-D and HAM-A were used to assess depression and anxiety levels of the participants at the time of the study. They are reliable and clinically valid rating scales that are widely used in practice (20-22) HAM-D and HAM-A are not suitable for diagnosing anxiety or depression; they are used for assessing the participant's mood related to anxious and depressive feelings (20-22) Therefore, no cutoff score was set, and outcomes of HAM-A and HAM-D were used numerically for statistical analysis.

\subsection{Sociodemographic data}

Participants were asked to fill out sociodemographic data forms along with CTQ, HAM-A, and HAM-D. Data on age, height, weight, and alcohol and cigarette use status were collected.

\subsection{Statistical analysis}

Statistical analysis was performed using the SPSS 15.0 software. The normality of the distributions was determined using visual graphs (e.g., histogram) and appropriate statistical methods. Student's t-test was used to compare the mean of the two groups with normal distribution, and MannWhitney $U$ test was used to compare the two groups without normal distribution. Kruskal-Wallis test was used to compare more than two groups with an abnormal distribution. MannWhitney $U$ test was used for post hoc comparison of the groups. Bonferroni correction was made when the MannWhitney $U$ test was used in post hoc comparisons. Chisquare test was used to compare categorical data. Spearman correlation analysis was performed to test the association between the two ranked variables. A $P$ value $<0.05$ was accepted as statistically significant.

\section{RESULTS}

There were 87 patients in both the study and the control groups with a total of 174 patients. The mean ages of the study and control groups were $36.8 \pm 12.4$ and $37.0 \pm 11.8$, respectively. There were $76(87.4 \%)$ females in the study group and $74(85.1 \%)$ females in the control groups. There was no statistically significant difference between the two groups regarding age $(P=0.935)$ and gender $(P=0.660)$ (Table 1$)$.
The mean body mass indices (BMI) of the study and control groups were $26.4 \pm 7.3$ and $24.9 \pm 3.8$, respectively. There was no statistically significant between the two groups $(P=0.097)$ (Table 1).

Of the groups, $29.9 \%$ of the study group was smoking regularly, whereas $21.8 \%$ of the control group had the same ratio. The alcohol dependency rates of the study and control groups were $6.9 \%$ and $1.1 \%$, respectively. However, there was no statistically significant difference in the cigarette and alcohol use rates between the two groups $(P=0.226$ and $\mathrm{P}=0.117$, respectively) (Table 1 ).

Table 1. Sociodemographic data of the study and control groups.

\begin{tabular}{|l|l|l|l|}
\hline & Study group & Control group & $\mathbf{P}$ \\
\hline Age (years) & $36.8 \pm 12.4$ & $37.0 \pm 11.8$ & $\mathrm{~F}=0.338, \mathrm{P}=0.935^{*}$ \\
\hline BMI $\left(\mathrm{kg} / \mathrm{m}^{2}\right)$ & $26.4 \pm 7.3$ & $24.9 \pm 3.8$ & $\mathrm{~F}=24.31, \mathrm{P}=0.097^{*}$ \\
\hline Gender (female) & $87.4 \%$ & $85.1 \%$ & $\chi^{2}=1.193, \mathrm{P}=0.660^{* *}$ \\
\hline Cigarette (use) & $29.9 \%$ & $21.8 \%$ & $\chi^{2}=1.469, \mathrm{P}=0.226^{* *}$ \\
\hline Alcohol (use) & $6.9 \%$ & $1.1 \%$ & $\chi^{2}=2.382, \mathrm{P}=0.117^{* *}$ \\
\hline
\end{tabular}

*Independent sample t-test.

**Chi-square test.

***Fisher's exact test.

As previously mentioned, we categorized participants into three clustered childhood trauma pattern groups using the CTQ. Healthy controls consisted of $55.8 \%$ of pattern group 1 and $55 \%$ of pattern group 2 . However, all of the participants who were in pattern group 3 had major depressive disorder (Table 2).

Table 2. Number of study and control participants in different pattern groups.

\begin{tabular}{|l|l|l|l|l|}
\hline & Pattern group 1 & Pattern group 2 & Pattern group 3 & Total \\
\hline Study $(n)$ & 34 & 36 & 17 & 87 \\
\hline Control $(n)$ & 43 & 44 & 0 & 87 \\
\hline
\end{tabular}

Anxiety and depression levels, which were assessed by HAM-A and HAM-D, respectively, both had positive correlations with emotional, physical, and sexual abuse (Table 3).

Table 3. Anxiety and depression levels in different classifications of childhood abuse and neglect.

\begin{tabular}{|l|l|l|l|l|l|}
\hline & $\begin{array}{l}\text { Emotional } \\
\text { abuse }\end{array}$ & $\begin{array}{l}\text { Emotional } \\
\text { neglect }\end{array}$ & $\begin{array}{l}\text { Physical } \\
\text { abuse }\end{array}$ & $\begin{array}{l}\text { Physical } \\
\text { neglect }\end{array}$ & $\begin{array}{l}\text { Sexual } \\
\text { abuse }\end{array}$ \\
\hline \multirow{2}{*}{ HAM-A } & $\mathrm{rs}=0.321$ & $\mathrm{rs}=0.050$ & $\mathrm{rs}=0.292$ & $\mathrm{rs}=-0.050$ & $\mathrm{rs}=0.225$ \\
\cline { 2 - 6 } & $\mathrm{P}<0.001$ & $\mathrm{P}=0.511$ & $\mathrm{P}<0.001$ & $\mathrm{P}=0.516$ & $\mathrm{P}=0.003$ \\
\hline \multirow{2}{*}{ HAM-D } & $\mathrm{rs}=0.371$ & $\mathrm{r} s=0.065$ & $\mathrm{rs}=0.360$ & $\mathrm{rs}=-0.026$ & $\mathrm{rs}=0.242$ \\
\cline { 2 - 6 } & $\mathrm{P}<0.001$ & $\mathrm{P}=0.396$ & $\mathrm{P}<0.001$ & $\mathrm{P}=0.731$ & $\mathrm{P}=0.001$ \\
\hline
\end{tabular}

The only two differences between the inclusion criteria for pattern groups 2 and 3 are the cut-off scores for physical and sexual abuse. However, as shown in Table 4, all median 
(minimum-maximum) CTQ scores of pattern group 3 were higher than those of pattern group 2. After post hoc analysis, we found that there was no statistically significant difference between pattern groups 2 and 3 only in physical neglect $(P=0.058)$. There was a statistically significant difference between pattern groups 2 and 3 in emotional neglect $(P=0.005)$ and all other comparisons between any of the two groups in all of the five main categories in CTQ $(P<0.001)$.

Table 4. Median (minimum-maximum) CTQ scores of participants in different pattern groups.

\begin{tabular}{|l|l|l|l|l|l|}
\hline & $\begin{array}{l}\text { Emotional } \\
\text { abuse }\end{array}$ & $\begin{array}{l}\text { Emotional } \\
\text { neglect }\end{array}$ & $\begin{array}{l}\text { Physical } \\
\text { abuse }\end{array}$ & $\begin{array}{l}\text { Physical } \\
\text { neglect }\end{array}$ & $\begin{array}{l}\text { Sexual } \\
\text { abuse }\end{array}$ \\
\hline Pattern group 1 & $5(5-7)$ & $8(5-12)$ & $5(5-5)$ & $5(5-7)$ & $5(5-5)$ \\
\hline Pattern group 2 & $7(5-17)$ & $14(5-25)$ & $5(5-9)$ & $8(5-16)$ & $5(5-7)$ \\
\hline Pattern group 3 & $11(5-24)$ & $19(6-24)$ & $12(5-16)$ & $10(5-14)$ & $8(5-14)$ \\
\hline P & $<0.001$ & $<0.001$ & $<0.001$ & $<0.001$ & $<0.001$ \\
\hline
\end{tabular}

Among patients with major depressive disorder, male $(n=11)$ participants had a mean score of 5 (minimum 5 and maximum 11) on the sexual abuse category of CTQ. Female $(n=76)$ participants also had a mean score of 5 (minimum 5 and maximum 14) in the same category. There was no statistically significant difference between the two genders $(P=0.902)$.

\section{DISCUSSION}

Our hypothesis was that children who experienced childhood trauma, especially physical and/or sexual abuse, are more susceptible to major depressive disorder. To test our postulate, we used the clustered childhood trauma pattern groups suggested by Schilling et al. (19). Participants in pattern group 1 had no trauma history. Pattern group 2 consisted of participants who have experienced significant emotional abuse, emotional neglect, and/or physical neglect and encountered mild to no physical or sexual abuse. Finally, pattern group 3 included participants with a history of physical and/or sexual abuse.

Just as we hypothesized, the pattern group that included participants with a history of physical or sexual abuse consisted solely of patients with depression; approximately $55 \%$ of pattern groups 1 and 2 comprised healthy controls.

It is evident from our results and previous studies that a history of physical and sexual abuse drastically increases the frequency of major depressive disorder. However, attributing the entire risk of depression to separate incidents of molestation would be an over-simplification. In pursuit of finding an explanation for this argument, we first investigated the correlation between five different categories of childhood trauma, as used in the CTQ, and severity of depression and anxiety.

We found that anxiety and depression levels, which were assessed by HAM-A and HAM-D, respectively, both had positive correlations with emotional, physical, and sexual abuse. However, there were no statistically significant correlations between HAM-A and HAM-D scores and emotional or physical neglect. This result was similar to various previous studies (22). Poole et al. established that out of every adverse childhood events, emotional abuse escalates the risk of major depressive disorder the most, by a factor of 3.5. In the same study, it is argued that emotional abuse is instrumental in the development of depressive cognitions (22).

This result only showed that emotional abuse also increased the risk of major depressive disorder, as an isolated incident. However, we argued that childhood traumas should not be considered as the sum of isolated incidents but a pattern of relationship (19). The results described in the previous study did not provide us much information on interrelation patterns. We then compared median CTQ scores of participants in different pattern groups to examine the pattern of relationship.

Except for physical neglect, all the comparisons between pattern groups 2 and 3 for median CTQ scores in all categories were statistically significant. These results supported our argument that isolated physical or sexual abuse incidents were not the only stressful events attributed to increasing the risk of depression. On top of the traumatic effects of physical and sexual abuse, they also indicate a defective pattern of emotional relationship. Median CTQ scores of pattern group 3 for emotional neglect and abuse were significantly higher than those of any of the other two pattern groups.

Stress and trauma usually are used in the same context; however, there is a distinct difference between the two. Stress is a disturbing life event, and (psychological) trauma is a distressing or disturbing experience. In other words, stress is an unprejudiced event, but trauma is the experience of the stressful incident.

CTQ is a tool that measures an individual's perception of their childhood traumas rather than actual stressful events, especially in other categories than physical and sexual abuse. Therefore, our data is limited to distinguish whether our results (median CTQ scores of pattern group 3 for emotional neglect and abuse being significantly higher than the other two pattern groups) were a consequence of an abused child's perception or inappropriate emotional relationship of a sexual and/or physical abuser. To provide a better insight into this debate, we plan to include the parents of the participants in our future studies.

If we investigate the limitations of our study further, psychiatric family histories were not included in our study. Our study only included patients who were admitted to our outpatient clinic, making our study susceptible to Berksonian bias. Since CTQ is a self-report questionnaire, self-report bias is another possible limitation of our study. Moreover, psychological resilience is another confounding factor, which is discussed in depth below.

Psychological resilience is another major factor that affects the clinical outcome of childhood trauma (22-24). Some 
studies established the impact of adaptive strategies on specific types of traumas. For example, emotional abuse has shown correlation with maladaptive emotion regulation, and emotional neglect has been associated with deficient adaptive regulation (24). For eliminating the confounding effect of psychological resilience, we plan to add appropriate tools, such as Connor-Davidson Resilience Scale or Cognitive Emotion Regulation Questionnaire, to our future studies.

Bulbul et al. established in their study that childhood trauma increases the risk of recurrent depressive attacks (25). History of childhood abuse or trauma of a major depressive patient, especially if there are repetitive depressive periods, should be thoroughly investigated for better treatment. Such thorough investigation would not only help us to prescribe an adequate psychopharmacological agent but also help us to set more suitable psychotherapeutic goals for the patient.

\section{DISCUSSION}

In conclusion, to provide a better idea of childhood trauma, we should not evaluate the clinical outcome as the sum of isolated incidents but as a defective pattern of relationship. A relationship containing physical and/or sexual abuse is expected to be distinctly different from the rest. Physical and sexual abuse apparently increases the risk of depression on their own, but they also indicate an amplified risk of experiencing emotional neglect and abuse, which is another risk factor for major depressive disorder.

Conflict of Interest: The authors have no conflict of interest to declare.

Financial Disclosure: No financial support.

\section{REFERENCES}

[1] World Health Organization. The world health report 2001-mental health: new understanding, new hope. World Health Organization. Available from: http://www.who.int/ whr/2001/en/whr01_en.pdf?ua=1. Published October 2001. Accessed 10.5.2017.

[2] Topuzoglu A, Binbay T, Ulas H, Elbi H, Aksu Tanik F, Zagli N, Alptekin $\mathrm{K}$. The epidemiology of major depressive disorder and subthreshold depression in Izmir, Turkey: Prevalence, socioeconomic differences, impairment and help-seeking. J. Affect. Disord. 2015; 181: 78-86.

[3] Rehan W, Antfolk J, Johansson A, Santtila P. Do single experiences of childhood abuse increase psychopathology symptoms in adulthood? J. Interpersonal Violence 2016; 1-18.

[4] Mulvihill D. The health impact of childhood trauma: An interdisciplinary review, 1997-2003. Issues Compr. Pediatr. Nurs. 2005; 28 (2): 115-36.

[5] Simonic E, Kastelan M, Peternel S, Pernar M, Brajac I, RončevićGržeta I, Kardum I. Childhood and adulthood traumatic experiences in patients with psoriasis. J. Dermatol. 2010; 37: 793-800.
[6] Cahill SP, Pontoski K. Post-Traumatic stress disorder and acute stress disorder I: Their nature and assessment considerations. Psychiatry (Edgmont). 2005; 2 (4): 14-25.

[7] Van Velzen LS, Schmall L, Jansen R, Milaneschi Y, Opmeer E, Elzinga BM, van der Wee N, Veltman D, Penninx B. Effect of childhood maltreatment and brain-derived neurtropic factor on brain morphology. SCAN 2016; 1841-1852.

[8] Di lorio CR, Carey CE, Michalski LJ, Corral-Frias NS, Conley ED, Hariri AR, Bogdan R. Hypothalamic-pituary-adrenal axis genertic variation and early stress moderates amygdala function. Psychoneuroendocrinol. 2017; 80: 170-178.

[9] Carr CP,Martins CM, Stingel AM, Lemgruber VB, Juruena MF. The role of early life stress in adult psychiatric disorders: a systematic review according to childhood trauma subtypes. J. Nerv. Ment. Dis. 2013; 201 (12): 1007-1020.

[10] Polat O. Tüm boyutlarıyla çocuk istismarı: Tanımlar 1. 2007; Hukuk Kitapları Dizisi: 782 (Turkish)

[11] Sar V, Ozturk E, Ikikardes E. Validity and reliability of the Turkish version of childhood trauma questionnaire. J. Med. Sci. 2012; 32 (4): 1054-1063.

[12] Senkal I. The investigation of the mediator role of alexithymia on the relationship of childhood traumas and attachment style with depression and anxiety symptoms in university students. Master's Thesis; Hacettepe University Social Sciences Institute, Ankara 2013.(Tukish)

[13] Turk S. Research of teacher candidates and teachers works on primary school in terms of child abuse potential. Master's Thesis; ZKU Institute of social sciences, Department of Educational Programs and Teaching 2010.

[14] Runyan D, Wattam C, Ikeda R, Hassan F, Ramiro L. World report on violence and health. Child abuse and neglect by parents and other caregivers. World Health Organization, Geneva (2002)

[15] Turhan E, Sangun O, Inandi T. Child abuse and prevention in primary care. STED 2006; 15 (9): 153.

[16] Taner YI, Gokler B. Çocuk istismarı ve inmali psikiyatrik yönleri. Hacettepe Tıp Dergisi 2004; 35: 82-86. (Turkish)

[17] Topbaş M. İnsanlı̆ı̆n en büyük ayıbı: çocuk istismarı. TSK Koruyucu Hekimlik Bülteni 2004; 3: 76-80. (Turkish)

[18] Norman RE, Byambaa M, De R, Butchart A, Scott J, Vos T. The long-term health consequences of child physical abuse, emotional abuse, and neglect: a systematic review and metaanalysis. PLOS Medicine 2012; 9 (11).

[19] Schilling C, Weidner K, Brähler E, Glaesmer H, Häuser W, Pöhlmann K. Patterns of childhood abuse and neglect in a representative German population sample. PLOS ONE 2016; 7 (11).

[20] Akdemir A, Turkcapar MH, Orsel SD, Demirergi N, Dag I, Ozbay $\mathrm{MH}$. Reliability and validity of the Turkish version of the Hamilton Depression Rating Scale. Compr. Pyschiatry 2001; 42 (2): 161-165.

[21] Aydemir O, Kirkpinar I, Sati T, Uykur B, Cengisiz C. Reliability and validity of the Turkish version of the health anxiety inventory. Arc. Neuropsychiatry 2013; 50 (4): 325-331.

[22] Poole JC, Dobson KS, Pusch D. Childhood adversity and adult depression: The protective role of psychological resilience. Child Abuse \& Neglect 2017; 64: 89-100.

[23] Ding H, Han J, Zhang M, Wang K, Gong J, Yang S. Moderating and mediating effects of resilience between childhood trauma 
and depressive symptoms in chinese children. J. Affect. Disord. 2017; 211: 130-135.

[24] Huh HJ, Kim KH, Lee H-K, Chae J-H. The relationship between childhood trauma and the severity of adulthood depression and anxiety symptoms in a clinical sample: The mediating role of cognitive emotion regulation strategies. J. Affect. Disord. 2017; 213: 44-50.

[25] Bulbul F, Çakır U, Ulku C, Ure I, Karabatak O, Alpak G. Childhood trauma in recurrent and first episode depression. Anatolian Psychiatry J. 2013; 14: 93-99. 